\title{
Evidence of charge localization from photoinduced infrared absorption in $\mathrm{BaBiO}_{3}$
}

\author{
C TALIANI ${ }^{\dagger}$, A J PAL, G RUANI, R ZAMBONI and \\ R MASINI* \\ Istituto di Spettroscopia Molecolare, CNR, Via de'Castagnoli 1, 40126 Bologna, Italy \\ *Istituto ITM, CNR, Via Bassini 15a, 20133 Milano, Italy
}

\begin{abstract}
Photoinduced infrared activities were studied in $\mathrm{BaBiO}_{3}$ and the results compared with those of $\mathrm{La}_{2} \mathrm{CuO}_{4}$ and $\mathrm{YBa}_{2} \mathrm{Cu}_{3} \mathrm{O}_{6+x}$. The spectral range of investigation covered the interval from 50 to $4000 \mathrm{~cm}^{-1}$. Clear evidence of lattice relaxation around the photoinjected charges was observed in the phonon spectral range with the bleaching of IR phonon bands. On the high energy side, we observed the rising edge of a broad electronic absorption. The results give an evidence of the suppression of the charge density wave due to the lattice relaxation around the photoinjected carriers. Moreover the low-energy charge excitation in the near infrared appears as a common characteristic of both layered and three-dimensional high $T_{c}$ superconductors, and may be relevant in the pairing mechanism.
\end{abstract}

Keywords. Superconductivity; charge density wave; optical properties.

\section{Introduction}

The widening of the chemical structures that support high $T_{c}$ superconductivity offers an interesting experimental and theoretical opportunity to verify the common characteristics which underlie all the systems and may eventually lead to the understanding of the mechanism of superconductivity.

It has been shown that the photoinjection of electron-hole pairs in $\mathrm{La}_{2} \mathrm{CuO}_{4}(\mathrm{Kim}$ et al 1987; Ginder et al 1988) and $\mathrm{YBa}_{2} \mathrm{Cu}_{3} \mathrm{O}_{6+x}(\mathrm{Kim}$ et al 1988; Taliani et al 1988, 1989) results in the formation of local electronic states which are associated with lattice distortions. These electronic excitations have been indicated as the pairing factors in the mechanism of high $T_{c}$ superconductivity.

In the quest of a general behaviour, we have investigated the semiconducting $\mathrm{BaBiO}_{3}$ material which is the precursor of the high $T_{c} \mathrm{Ba}_{1-x} \mathrm{~K}_{x} \mathrm{BiO}_{3}$ compound $\left(T_{c}=30 \mathrm{~K}\right.$ ) (Mattheis et al 1988; Cava et al 1988). This compound is interesting for the following reasons: (i) is the only one without $\mathrm{Cu}-\mathrm{O}$ planes, (ii) has no magnetic ions, (iii) is three-dimensional rather than layered and (iv) shows a static charge density wave (CDW) in the insulating phase up to the insulator-metal transition. In this respect it is useful to compare the optical properties of $\mathrm{BaBiO}_{3}$ with those of the conventional Cu-oxide high $T_{c}$ superconductors by the photoinduced absorption technique.

\footnotetext{
${ }^{+}$For correspondence.
} 


\section{Experiment}

\subsection{Sample preparation}

Stoichiometric proportions of powdered metal nitrates dissolved in distilled water were employed to synthesize $\mathrm{BaBiO}_{3}$ by the citrate-polymer route (Wang et al 1987). The resulting solution was stirred up and heated to $90^{\circ} \mathrm{C}$, and let react. At complete reaction the resulting gel was subsequently decomposed to a solid by heating. The powders were calcined at $450^{\circ} \mathrm{C}$ and then ground, pressed into pellets, sintered and annealed at $950^{\circ} \mathrm{C}$. X-ray diffraction measurements showed that the product was $X$-ray pure (Thornton and Jacobson 1978).

Pressed pellets were used for the optical spectroscopic experiments. The ceramic was ground and mixed with polyethylene or CsI powders $(0.5-4.0 \mathrm{wt} \%)$ in an agate mortar and then pressed to obtain optically transparent pellets in the far-IR and mid-IR spectral range respectively.

\subsection{Experimental set-up}

Absorption spectra and photoinduced absorption (PA) measurements were performed in a cryostat at $10 \mathrm{~K}$ by using a modified Bruker FTIR interferometer; an $\mathrm{Ar}^{+}$laser was used for photoexcitation at $2.5 \mathrm{eV}$. Consecutive interferograms of 32 scans each, with laser on and off, were stored for a total number of about 5000 scans in order to achieve an appreciable $\mathrm{S} / \mathrm{N}$ ratio. The $\mathrm{PA}$ spectrum was derived by calculating the $-\Delta T / T$ ratio (i.e. the fractional variation of transmittance due to photoinduction).

\section{Results}

\subsection{Infrared absorption}

The absorption spectrum of $\mathrm{BaBiO}_{3}$ (figure 1) shows four bands in the phonon spectral range.

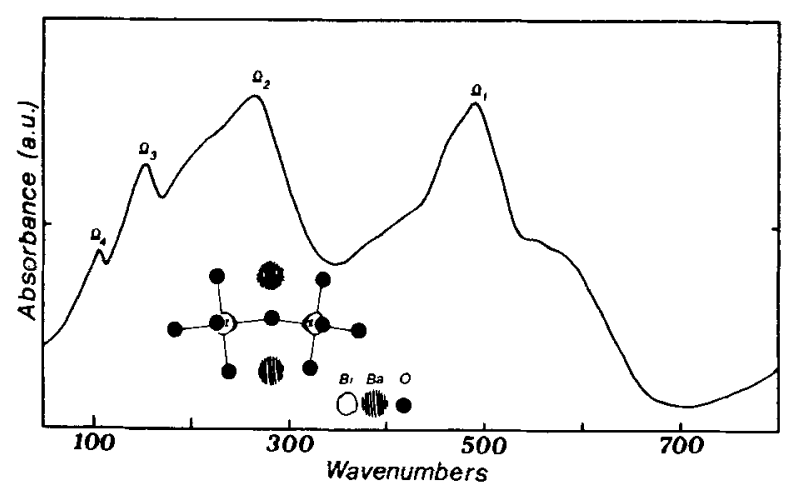

Figure 1. Infrared absorption spectra of $\mathrm{BaBiO}_{3}$. Inset is the monoclinic $\mathrm{BaBiO}_{3}$ structure. 
This is consistent with the monoclinic distortion induced by the coupling of the lattice with a $\mathrm{CDW}$ (there are two inequivalent $\mathrm{BiO}_{3}$ octahedra with the consequent doubling of the cell, see inset in figure 1). This dimerization results in the activation of an acoustic mode in the cubic undimerized structure, that becomes IR-active (TO $\left.{ }_{4}^{\prime}\right)$ because of the consequent folding of the Brillouin zone. Uchida et al (1985) proposed the following assignment of the phonons: $\Omega_{1}$ at $492 \mathrm{~cm}^{-1}$ is the bending mode of the $\mathrm{BiO}_{3}$ octahedra, $\Omega_{2}$ at $264 \mathrm{~cm}^{-1}$ is the $\mathrm{Bi}-\mathrm{O}$ stretching mode, $\Omega_{3}$ at $155 \mathrm{~cm}^{-1}$ is the $\mathrm{TO}_{4}^{\prime}$ mode and the $\Omega_{4}$ at $104 \mathrm{~cm}^{-1}$ is the external mode (the octahedra are moving as a whole).

\subsection{Photoinduced absorption}

3.2a Phonon modes: The phonon band at $492 \mathrm{~cm}^{-1}$ assigned at $\Omega_{1}$ is clearly affected by photoexcitation. This band presents a structured photoinduced bleaching (PB) suggesting that some components of the absorption band are bleached to a different extent. In view of this, we investigated the laser intensity dependence $\left(\boldsymbol{I}_{\text {laser }}\right)$ of the total bleached area $\left(A_{\mathrm{PB}}\right)$ where:

$$
A_{\mathrm{PB}}=\int_{\Omega_{1}}(\Delta T / T)_{l_{\text {laser }}} \mathrm{d} \Omega .
$$

$A_{\mathrm{PB}}$ varies proportionally to the square root of $\boldsymbol{I}_{\text {laser }}$ as shown in the inset of figure 2 . Such a dependence excludes also the possibility of a thermal effect. A decrease of the

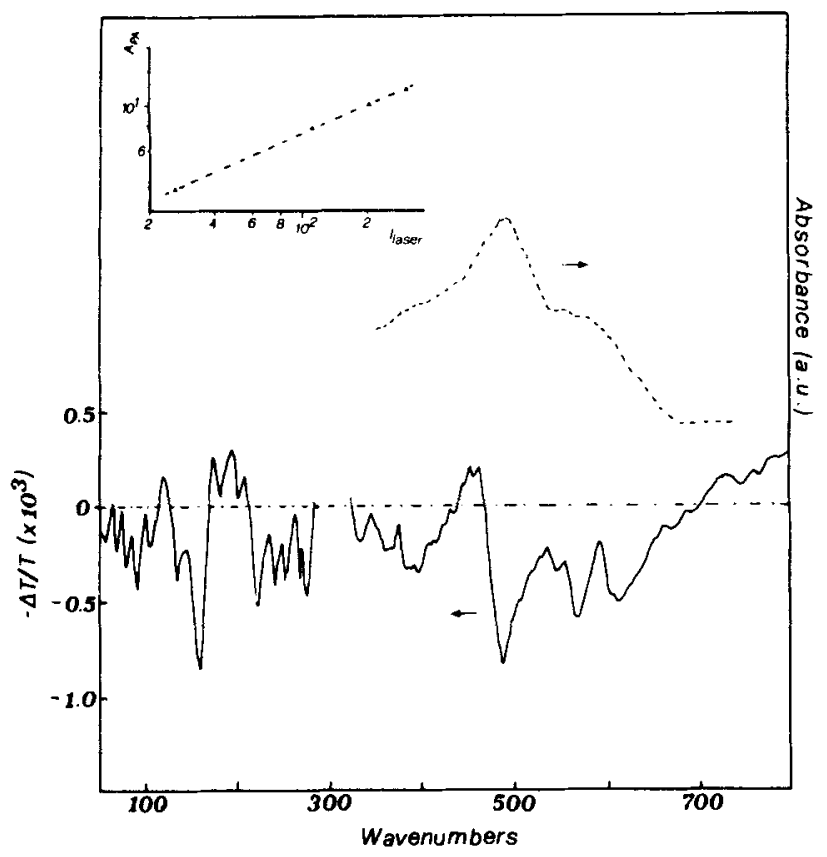

Figure 2. Photoinduced absorption spectrum of $\mathrm{BaBiO}_{3}$ at $10 \mathrm{~K}$ of phonon region (continuous line), detailed view of the $500 \mathrm{~cm}^{-1}$ IR band (dashed line). Inset is the laser intensity $\left(\mathrm{mW} / \mathrm{cm}^{-2}\right)$ dependence of the effective bleached area of mode $\Omega_{1}$. 
oscillator strength of this band has been observed by Uchida et al (1985) with the increase of $\mathrm{Pb}$ doping in $\mathrm{BaBiO}_{3}$.

Other bleachings appear in correspondence with the $\Omega_{2}$ and $\Omega_{3}$ bands, but we cannot exclude that thermal effects contribute to these features. Nevertheless in the thermal modulation spectra the bleaching of $\Omega_{3}$ is weaker than the bleaching of $\Omega_{2}$ contrary to what we observe in PA spectra (see figure 2). From these considerations we tend to believe that the photoinduced bleaching of $\Omega_{3}$ is intrinsic.

3.2a Charge excitations: In figure 3 the photoinduced spectrum is shown up to $4000 \mathrm{~cm}^{-1}$. A broad absorption envelope with an onset at $1000 \mathrm{~cm}^{-1}(0.12 \mathrm{eV})$ increases in intensity towards the high energies and levels off at about $4000 \mathrm{~cm}^{-1}$ $(0.5 \mathrm{eV})$. Due to the remarkable oscillator strength underlying the absorption we assign this feature to a dipole allowed electronic transition. This feature corresponds with a photoinduced electronic band with maximum at $0.5 \mathrm{eV}$ observed by photomodulation spectroscopy (Wei et al 1990). The laser intensity dependence at $2800 \mathrm{~cm}^{-1}$ is proportional to $I_{\text {laser }}{ }^{1 / 2}$ as in the case of the $\Omega_{1}$ phonon band bleaching.

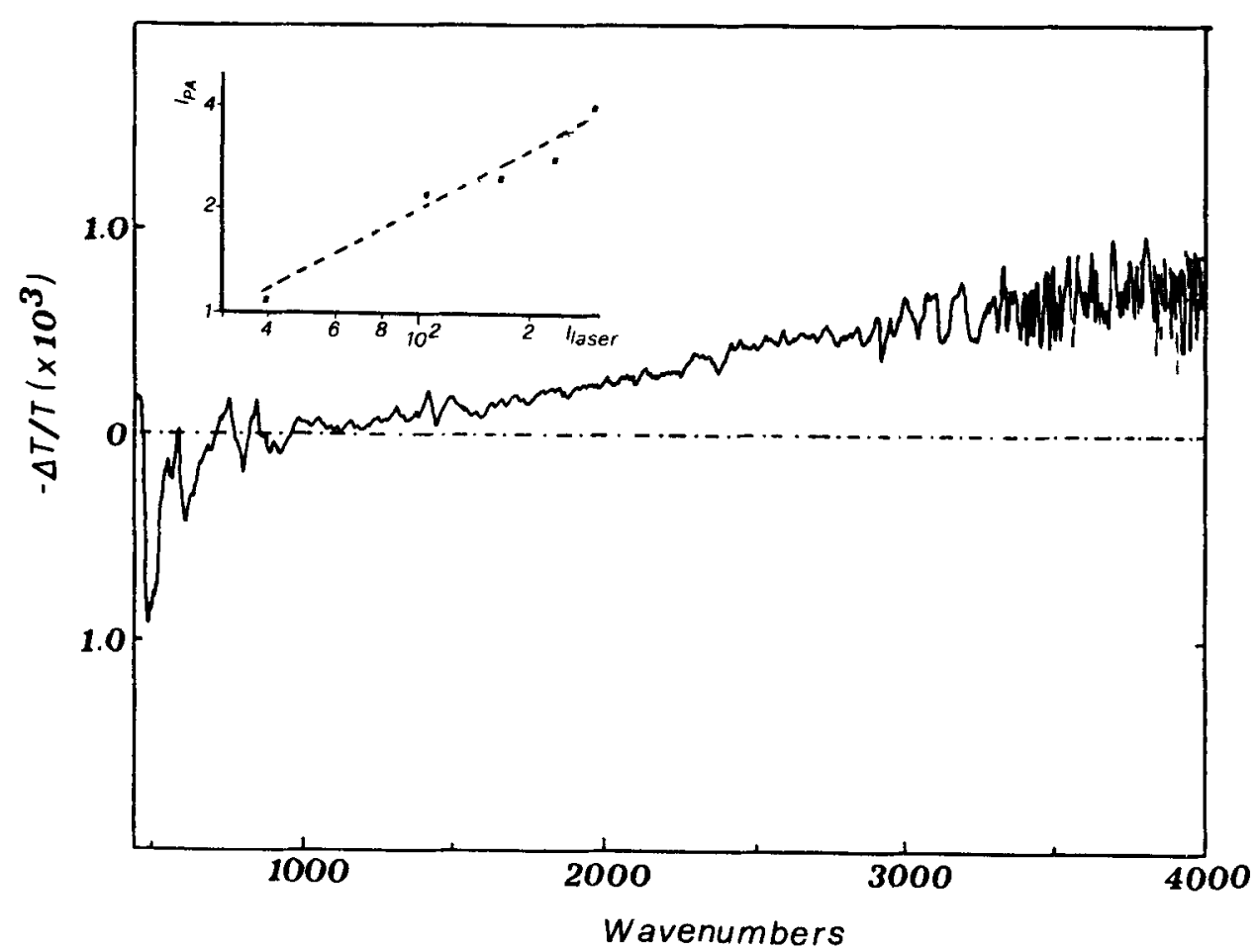

Figure 3. Photoinduced absorption spectrum of $\mathrm{BaBiO}_{3}$ at $10 \mathrm{~K}$. Inset is the laser intensity $\left(\mathrm{m} \mathrm{W} / \mathrm{cm}^{-2}\right.$ ) dependence of the electronic PA. 


\section{Discussion}

As has been observed in layered cuprate semiconducting precursors of the HTSC materials, photoexcitation of $\mathrm{BaBiO}_{3}$ leads to the generation of electron-hole pairs and eventually gives rise to a lattice distortion around the photoinjected charges which is accompanied by a modification of the electronic structure.

In the case of $\mathrm{BaBiO}_{3}$, where the electronic gap at the Fermi energy is due to the doubling of the unit cell due to the CDW (Mattheiss and Hamman 1983), the photoinjected charges reduce the CDW order parameter inducing a local electronic state within the gap. The photobleaching of the $\Omega_{3}$ phonon mode, which is made IR-active by the CDW distortion, is also a strong indication of the local suppression of the CDW due to the photoinduced carriers.

In $\mathrm{BaBiO}_{3}$, contrary to $\mathrm{La}_{2} \mathrm{CuO}_{4}$ and $\mathrm{YBa}_{2} \mathrm{Cu}_{3} \mathrm{O}_{6+x}$ where $A_{9}$ phonon modes are made IR-active, no photoinduced phonon band appears. This is consistent with the fact that the symmetry of the undimerized structure of $\mathrm{BaBiO}_{3}$ does not allow the activation of any $A_{g}$ mode in Raman scattering.

It is interesting to notice that both the electronic PA band and the PB of the $\Omega_{1}$ mode have the same laser power dependence. The power $1 / 2$ dependence is a typical indication of a bimolecular recombination process of the photogenerated species. This implies that the decay rate is proportional to $n_{\mathrm{ps}}^{2}$, where $n_{\mathrm{ps}}$ is the number of photoexcited species. Considering a generation rate of these species proportional to the laser intensity, we can write the rate equation for $n_{\mathrm{ps}}$ as:

$$
\mathrm{d} n_{\mathrm{ps}} / \mathrm{d} t=\alpha I_{\text {laser }}-\beta n_{\mathrm{ps}}^{2}
$$

where $\alpha$ and $\beta$ are constants. In steady-state conditions $\left(\mathrm{d} n_{\mathrm{ps}} / \mathrm{d} t=0\right)$ the number of photoexcited species is proportional to the square root of the illumination intensity:

$$
n_{\mathrm{ps}}=\alpha / \beta I_{\text {laser }}^{1 / 2}
$$

This dependence indicates that the photogenerated carriers recombine in pairs. The behaviour differs from the conventional semiconductors in which the recombination is monomolecular because of its defect-assisted nature and hence the recombination is determined by the density of these defects and not by the density of photoexcited species. Our experimental method allows detection of only long lived photoexcited species, with lifetime in the order of milliseconds. The exclusion of a defect-trapping process indicates that the long lifetime is not due to defect states but rather that the photoexcited species are indeed self-trapped.

This is indicative of a strong interaction between the lattice and charge carriers. The similar square root dependence of the photoinduced charge excitation at $0.5 \mathrm{eV}$ and the phonon bleaching indicates that both these signatures are characteristics of the same photoexcited species. This behaviour is common for several superconducting systems including $\mathrm{La}_{2} \mathrm{CuO}_{4}, \mathrm{YBa}_{2} \mathrm{Cu}_{3} \mathrm{O}_{6+x}$ and thallium-based perovskites (Foster et al 1989) despite the different structures and basic compositions.

In this respect, the strong similarity between the mid-IR absorption derived from reflectivity measurements of the superconductors and the photoinduced absorption of the parent semiconductors is noticeable. The common low-energy photoinduced charge excitation may be relevant for the pairing mechanism. 


\section{Conclusions}

In summary, the photogenerated carriers interact strongly with phonons reducing locally the CDW distortion. This effect is accompanied by the creation of a new electronic state within the optical gap, and the modification of the selection rules of IR phonon absorption. This is a strong evidence of a substantial electron-phonon interaction. In particular, the low energy charge excitation, which is a common feature of all the high temperature superconductors, may play a fundamental role in pairing mechanisms.

\section{References}

Cava R J, Batlogg B, Krajewski J J, Farrow R, Rupp L W Jr, White A E, Peck W F and Kometani T 1988 Nature (London) 332814

Foster C M, Heeger A J, Kim H V, Stucky G and Herron N 1989 Synth. Met. 33171

Ginder J M, Roe M G, Song Y, McCall R P, Gaines J R, Ehrenfreund E and Epstein A 1988 Phys. Rev. B37 7506

Kim Y H, Heeger A J, Acedo L, Stucky G and Wudl F 1987 Phys. Rev. B36 7252

Kim Y H, Foster C M, Heeger A J, Cox S and Stucky G 1988 Phys. Rev. B38 6478

Mattheis L F and Hamman D R 1983 Phys. Rev. B28 6629

Mattheis L F, Gyorgy E M and Johnson D W 1988 Phys. Rev. B37 3745

Taliani C, Zamboni R. Ruani G, Matacotta F C and Pokhodnya K I 1988 Solid State Commun. 66487

Taliani C, Zamboni R, Ruani G, Pal A J, Matacotta F C, Vardeny Z and Wei X 1989 in Electronic structure of high $T_{\mathrm{c}}$ superconductors, (eds) A Bianconi and A Marcelli (Oxford: Pergamon Press) p. 95 Thornton G and Jacobson A J 1978 Acta Crystallogr. B34 351

Uchida S, Tajima S, Masaki A, Sugai S, Kitazawa K and Tanaka S 1985 J. Phys. Soc. Jpn. 544395

Wang $\mathrm{H} \mathrm{H}$ et al 1987 Inorg. Chem. 261476

Wei X, Vardeny Z V, Chen L, Taliani C, Zamboni R, Pal A J and Ruani G 1990 APS Meeting 